\title{
Are CT heads indicated in the primary investigation of acute psychosis?
}

\begin{abstract}
Aim: The aim of this study was to assess the pick-up of rate of organic brain abnormalities in patients who presented with first episode psychosis (FEP) and the impact of positive findings on clinical management.
\end{abstract}

Background: First episode psychosis refers to the first time person experiences psychotic symptoms. This includes people who have recently had their first psychotic episode but can also include those who have been treated for psychosis for some time but have not seen any improvement in their symptoms.

Keywords: psychosis, clinical management, psychotic symptoms, neurology, patients

\author{
Volume 4 Issue 2 - 2018 \\ R Hubbard,' G Sheehan-Dare, ${ }^{2}$ A Leigh, ${ }^{3} \mathrm{~S}$ \\ Cordell,' S Sciacca, ' A Higgit, ${ }^{4}$ N Khan, ${ }^{5} \mathrm{~F}$ \\ Kazmi ${ }^{5}$ \\ 'Radiology SpR, UK \\ ${ }^{2}$ Foundation year 2 Doctor, UK \\ 3Psychiatry CTI LAS, UK \\ ${ }^{4}$ Psychiatry Consultant, UK \\ ${ }^{5}$ Radiology Consultant, UK
}

Correspondence: R Hubbard, Radiology Department, Chelsea and Westminster Hospital, United Kingdom,

Email hubbsy@doctors.org.uk

Received: June 01, 2017| Published: April 27, 2018

\section{Introduction}

Amongst international guidelines, there is no general consensus about the routine use of cross-sectional imaging in the evaluation of patients with first episode psychosis. In the U.K, the guidance from
NICE recommends against the use of routine imaging in the absence of focal neurology. ${ }^{1}$ Guidelines in Australia and New Zealand, however, recommend that a baseline CT examination be obtained. At our local psychiatric hospital within West London, CT head scans are requested as a first line investigation in FEP (Table 1).

Table I The table below shows a comparison of the guidelines of neuro-imaging in FEP in the UK,American, Canadian and Australia/New Zealand

National Institute for Health and
Clinical Excellence (2008)
"Structural neuroimaging techniques
(either magnetic resonance imaging (MRI)
or computed axial tomography (CT)
scanning) are not recommended as a
routine part of the initial investigations
for the management of first-episode
psychosis."
“...this decision should not affect the
current practice of using structural
neuroimaging techniques selectively to
exclude organic causes of psychosis where
people's symptoms, or other aspects
of their presentation, suggest a higher
likelihood of an underlying organic cause."

Another study done in Portugal from 2016 called "Retrospective study in structural neuroimaging in first-episode psychosis" looked at retrospective data obtained from 32 patients aged between 18-48 years old who presented with FEP. CT and MRI images and their reports were analysed but none of the patients showed any abnormalities in their neuroimaging exams causing psychotic symptoms. $37 \%$ of patients had incidental findings on their neuroimaging, not related to psychosis, such as brain atrophy, dilated lateral ventricles and arachnoid cyst. This study concluded that "This study suggests that structural neuroimaging exams reveal scarce abnormalities in young patients with first-episode psychosis. Structural neuroimaging is

\section{Royal Australian and New Zealand College of Psychiatrists 2005 (McGorry, 2005)}

"Excluding patients with head injury, neurologic disease, seizures, or substance abuse, $7.9 \%$ of MRI scans obtained in first-episode patients were of "clinical importance, affecting prognosis, diagnosis, or management."

"Recommends CT or MRI in baseline assessment of first-episode psychosis."
Baseline MRI scan should be done as part of optimal initial assessment in firstepisode psychosis.

especially useful in first-episode psychosis patients with neurological symptoms, atypical clinical picture and old age".

Another paper written in 2013 titled "The role of MRI and CT of the brain in first episodes of psychosis" concluded that MRI was comparable with CT in terms of diagnosis of both pathological and incidental and cerebral lesions. ${ }^{2}$ This paper looked at 112 MRI scans and $204 \mathrm{CT}$ scans and found that $<3 \%$ of patients presenting with FEP had brain lesions potentially accountable for psychosis. Approximately $65 \%$ of patients had incidental brain lesions including cerebral atrophy, small vessel ischaemic changes, unruptured Circle of Willis aneurysms, cavernoma and arachnoid cysts. 
It concluded, "Routine MRI or CT imaging of the brain is unlikely to reveal disease leading to a significant change in management. MRI was comparable with CT in terms of diagnosis of both pathological and incidental cerebral lesions. Therefore, routine brain structural imaging of FEP in patients without focal neurology may not be routinely required and if imaging is requested then $\mathrm{CT}$ may function equally as well as MRI as the first-line investigation".

\section{Methods}

A retrospective review of all $\mathrm{CT}$ head scans performed on patients at The Gordon Hospital (West London) with FEP presenting between February 2014 and April 2017 was conducted. CT imaging requests and reports were identified and analysed using our local imaging system (PACS). Psychiatric entries from JADE were reviewed in

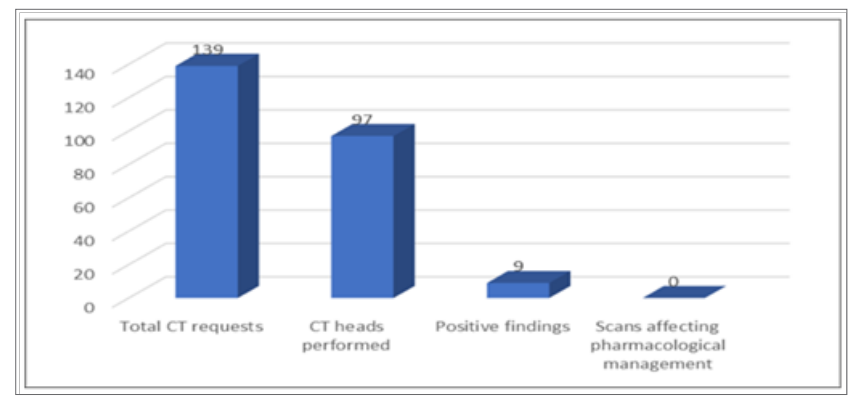

Figure I Number of scans requested.

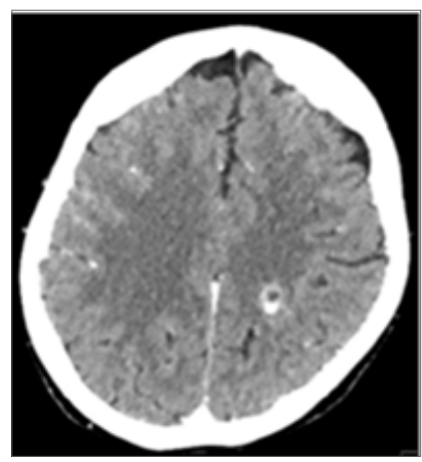

Figure 3 CT Head demonstrating a cavernous malformation.

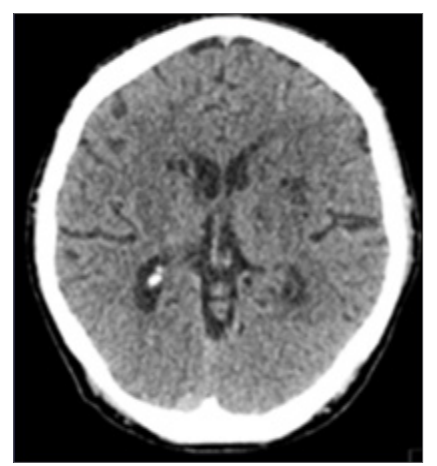

Figure 5 CT Head demonstrating superior vermis atrophy.

\section{Conclusion}

The results of our study demonstrate a $9 \%$ pick-up rate in line with patients where pathology on imaging was identified to ascertain any changes in clinical management. ${ }^{3}$

\section{Results}

$139 \mathrm{CT}$ head requests were identified and 97 were performed. 42 (33\%) patients did not attend for their CT head scans. The age range for our data set was 18-78 years old with a median age of 44 . The median age of the patients showing positive findings was 53 years old. None of the findings were of space occupying lesions, and despite one loss to follow up, no change to pharmacological management was made. Two patients were referred to neurosurgery for specialist opinion or counselling of bleeding risk. Two patients were referred to memory services due to atrophy seen on imaging in combination with a low ACE score (Figure 1-5).

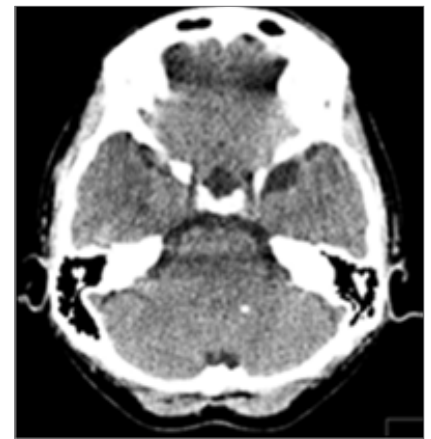

Figure 2 CT Head demonstrating calcification.

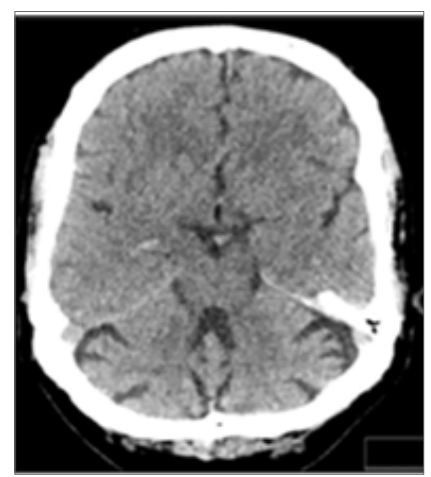

Figure 4 CT Head demonstrating lacunar infarcts.

national data. Pharmacological management of the patients with FEP remain unchanged in all those seen to follow up. Four referrals to other services were made on the basis of incidental findings as mentioned above, but half of these were in combination with results from other tools of psychiatric assessment (ACE proforma).

A high DNA rate of CT head scans has implications on cost and management for radiology departments. It may be that clinicians in mental health services are aware of the impact of health inequalities on their patients and are actively seeking to counteract them, however, in view of NICE guidelines and the low pick-up rate of positive findings, clinicians should consider the value of cross-sectional imaging in their practice in the management of patients with FEP. Of the studies performed, $9(9 \%)$ showed positive findings which included: deep white matter small vessel disease; lacunar infarcts; isolated focus/foci of calcification; development venous anomaly/ cavernous malformation; involutional change. 


\section{Acknowledgements}

None.

\section{Conflict of interest}

The author declares that there is no conflict of interest

\section{References}

1. http://guidance.nice.org.uk/TA136/Guidance/doc/English

2. Khandanpour N1, Hoggard N, Connolly DJ. The role of MRI and CT of the brain in first episodes of psychosis. Clin Radiol. 2013;68(3):245-250.

3. Coentre R, Silva-dos-Santos Amilcar, Talina MC. Retrospective study on structural neuroimaging in first-episode psychosis. Peer J. 2015;4:e2069. 